\title{
Originales
}

\section{Evaluación de la exposición laboral a plaguicidas en España mediante una matriz empleo-exposición (MatEmESp, 1996-2005)}

\author{
Assessment of occupational exposure to pesticides in Spain through a job- \\ exposure matrix (MatEmESp, 1996-2005)
}

\author{
Javier Vila ${ }^{1,2,5}$, Rudolf van der Haar ${ }^{1,3}$, Ana M. García ${ }^{1,4,5}$ \\ 1. Centro de Investigación en Salud Laboral (CiSAL). España. \\ 2. Universitat Pompeu Fabra (UPF). España. \\ 3. MC-MUTUAL. España. \\ 4. Departamento de Medicina Preventiva y Salud Pública, Universitat de València. España. \\ 5. CIBER de Epidemiología y Salud Pública (CIBERESP). España.
}

Recibido: 04-11-14

Aceptado: 18-11-14

\author{
Correspondencia \\ Javier Vila \\ CiSAL (Centro de Investigación en Salud Laboral). \\ C/ Dr. Aiguader 88 1. a planta, \\ 08003 Barcelona. España. \\ Telf. 932147 325| Fax 932147302 \\ Correo electrónico: javier.vila@upf.edu
}

Resumen

Introducción: La exposición laboral a plaguicidas ha sido asociada a numerosos problemas de salud. Sin embargo, la información sobre la exposición de los trabajadores afectados en España es escasa y dispersa.

Objetivos: El objetivo de este trabajo fue estimar el nivel y la prevalencia de la exposición laboral a plaguicidas en España para el periodo 1996-2005, como parte del proyecto de construcción de la matriz empleo-exposición española, MatEmESp.

Métodos: El establecimiento de las ocupaciones expuestas, los agentes de referencia y las estimaciones de exposición se basó en información de otras matrices, datos de evaluaciones de riesgos en empresas españolas y revisión de la bibliografía.

Resultados: De las 482 ocupaciones incluidas en la CNO-94, se identificaron 45 expuestas. Para fungicidas y herbicidas, en 39 ocupaciones se estimó un nivel bajo de exposición ( $\leq 5 \%$ VLA), y en otras 6 un nivel medio (6-25\%VLA). Para insecticidas, se estimó un nivel bajo de exposición en 19 ocupaciones y medio en las 26 restantes.

Conclusiones: Este trabajo representa la primera recolección sistemática de datos de exposición laboral a plaguicidas en España. Las estimaciones de exposición obtenidas están disponibles en la página web de MatEmESp (www.matemesp.org) y pueden ser útiles para la investigación y la gestión de las actividades preventivas en España.

Med Segur Trab (Internet) 2014; 60 (237) 645-659

Palabras clave: Matriz empleo-exposición, exposición laboral, plaguicidas, trabajadores. 


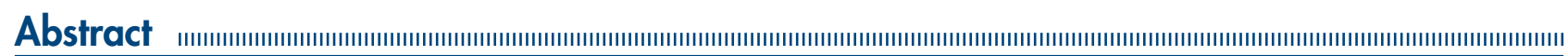

Background: Occupational pesticide exposure has been associated with different health problems. However, the available information on the characteristics of the exposure for the affected workers is sparse and scattered.

Objectives: This study aimed at estimating the level and prevalence of occupational pesticide exposure in Spain for the period 1996-2005, as part of the project to develop a general Spanish job-exposure matrix, MatEmESp.

Methods: Data from other matrices, risk assessments in Spanish companies and a literature review were used to identify exposed occupations and reference chemical agents as well as to establish the exposure estimates.

Results: Of the 482 occupations in the Spanish job coding system, 45 were identified as exposed. For fungicides and herbicides, 39 occupations showed a low level of exposure ( $\leq 5 \%$ OEL) whereas 6 were found to have a medium level (6-25 \% OEL). For insecticides, low exposure was found in 19 occupations while 26 showed a medium level.

Conclusion: This is the first systematic collection of data on occupational exposure to pesticides in Spain. The exposure estimates obtained, available at the MatEmESp web page (www.matemesp.org), can be useful for research and health and safety management programs in Spain.

Med Segur Trab (Internet) 2014; 60 (237) 645-659

Key words: Job-exposure matrix, occupational exposure, pesticides, workers. 


\section{INTRODUCCIÓN}

\section{Características de la exposición laboral a plaguicidas en España}

Un plaguicida es una sustancia o mezcla de sustancias destinada a prevenir, controlar o destruir plagas. Se denomina ingrediente o sustancia activa la parte biológicamente activa presente en una formulación ${ }^{1}$. Según su uso, se distinguen dos grandes grupos de plaguicidas, los fitosanitarios y los biocidas, cuya autorización y comercialización están reguladas en España por los RD 2163/1994 y 1054/2002, respectivamente. Los fitosanitarios se destinan a la protección de las cosechas y productos agrarios mientras que los biocidas se utilizan en el control de plagas no agrícolas o estructurales.

El uso laboral de plaguicidas se asocia a múltiples actividades entre las que destacan la agricultura, la jardinería y la silvicultura (fitosanitarios), así como la ganadería, los servicios de salud pública y gestión de plagas estructurales o el tratamiento de la madera (biocidas) $^{1}$. Según datos facilitados por la Asociación Empresarial para la Protección de las Plantas (AEPLA), en 2005 se consumían en España casi 100.000 toneladas/año de fitosanitarios. Para este mismo año, y según datos aportados por ADELMA (Asociación de Empresas de Detergentes y Productos de Limpieza), el consumo de biocidas ascendía a unas 2.000 toneladas/año ${ }^{2,3}$ (Figuras 1 y 2). A partir de esta fecha se produce una ligera disminución en el consumo de plaguicidas, de manera que, según los datos más recientes publicados por el Ministerio de Medio Ambiente sobre el consumo de fitosanitarios, el consumo total en 2012 fue de menos de 50.000 toneladas/año ${ }^{4}$.

Figura 1. Consumo de fitosanitarios en España 1996-2005 (Fuente AEPLA*)

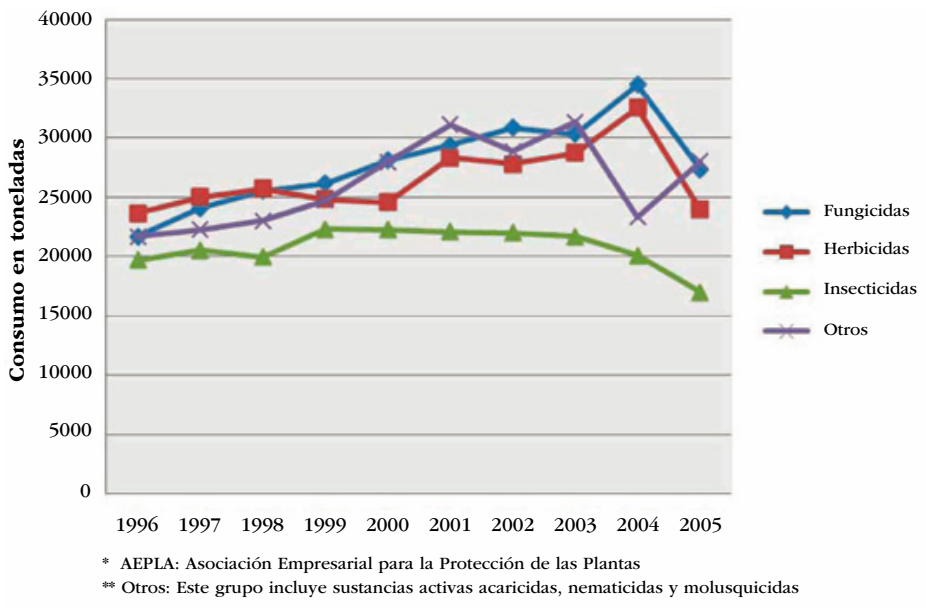

Figura 2. Consumo de biocidas en España 1996-2005 (Fuente: ADELMA*)

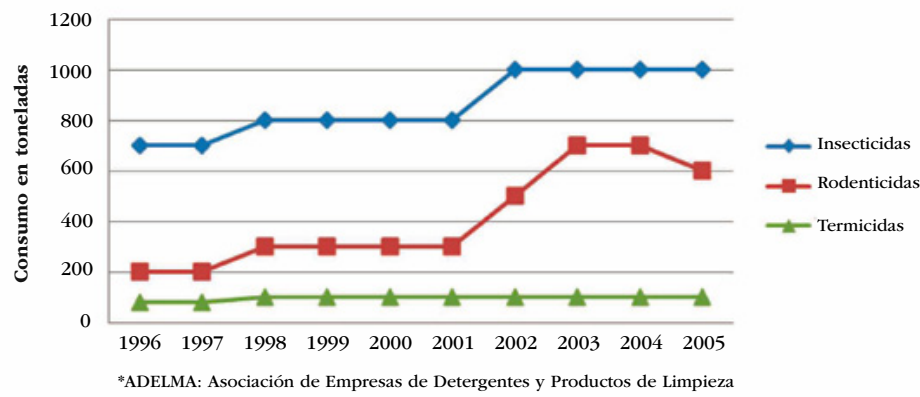

De acuerdo con la última encuesta nacional de condiciones de trabajo en el sector agropecuario realizada en España (2011), más del 50\% de los trabajadores en este sector (con más de 350.000 trabajadores en esta fecha) realizaba tratamientos con plaguicidas5. Algunos estudios ${ }^{6}$ sugieren que estos trabajadores no siempre están suficientemente 
formados sobre los riesgos derivados de la manipulación de plaguicidas, además de existir un uso muy deficiente de las medidas de protección individual.

La exposición a plaguicidas en el trabajo puede ser directa, afectando a los trabajadores que manipulan y/o aplican las diferentes formulaciones, o indirecta, a través de la cual pueden verse afectados los trabajadores en las proximidades de zonas de aplicación/manipulación o en contacto con zonas tratadas. En lo relativo a las vías de exposición, la vía dérmica y la inhalatoria son las más importantes, aunque la vía digestiva, asociada a comer, beber o fumar en el trabajo, puede llegar a tener importancia en la dosis total absorbida ${ }^{7,8}$.

Existen en España límites de exposición profesional, conocidos como valores límite ambientales (VLA), para los agentes químicos considerados como peligrosos de acuerdo con el RD 374/2001. En relación con los plaguicidas, se han establecido VLA para unas 80 sustancias activas. Casi la mitad de ellas incluyen además la notación "Vía dérmica", al considerarse que presentan un riesgo alto de absorción por esta vía 9 .

\section{Matrices empleo-exposición}

Las matrices empleo-exposición (MEE) son sistemas de información que permiten asociar códigos de ocupación o actividad económica, entre otros, con diferentes agentes o exposiciones de riesgo en el trabajo. En su forma más básica, una MEE está formada por un eje de ocupaciones (habitualmente codificadas siguiendo un sistema estandarizado) y otro de agentes (físicos, químicos, de seguridad, etc.) para un periodo y ámbito determinados. En el interior de la matriz (las «celdas») se encuentra la información relativa a las características de la exposición, por ejemplo en forma de estimaciones de prevalencia e intensidad de la exposición a cada agente y en cada ocupación.

Existen a nivel internacional matrices empleo-exposición con estimaciones de la exposición laboral a plaguicidas para distintas poblaciones laborales. Así, en 1998 el instituto finlandés de salud ocupacional (FIOH) desarrolló la primera matriz general para población laboral finlandesa (FINJEM) ${ }^{10}$, con estimaciones cuantitativas de exposición laboral para diversos agentes, incluidos los plaguicidas. En otros países, las matrices se refieren a actividades específicas, como es el caso de los Países Bajos, donde se creó una MEE con estimaciones de exposición para plaguicidas de uso agrícola (fitosanitarios) en función del tipo de cultivo ${ }^{11}$. En Francia, se elaboró en 2008 otra matriz específica para fitosanitarios, MATPHITO, dentro del proyecto MATGENE ${ }^{12}$, en el que se prevé el desarrollo de 18 MEE referidas a diferentes agentes específicos. En el Reino Unido se construyó en 2010 la matriz TEMPEST, también específica para plaguicidas ${ }^{13}$ y que incluye estimaciones de exposición a estos agentes en función de sus usos y tareas asociadas. La información en esta última matriz incluye estimaciones de exposición inhalatoria y dérmica a diversas sustancias activas para el periodo 1945-2005 y cubre múltiples ocupaciones, incluidas actividades no agrícolas.

En 2009 se elabora en España la primera matriz empleo-exposición general para población trabajadora española, MatEmEsp ${ }^{14,15}$, que incluye estimaciones de la exposición laboral a agentes y condiciones de trabajo en cinco categorías (higiene, seguridad, ergonomía, factores psicosociales y condiciones de empleo) para el periodo 1996-2005 y para cada una de las ocupaciones codificadas en la Clasificación Nacional de Ocupaciones en España en su versión de 1994 (CNO-94). Las estimaciones contenidas en MatEmESp se obtuvieron a partir de información contenida en otras MEE, y en encuestas, estudios y registros preferentemente referidos a la población trabajadora en España, con la participación de expertos y técnicos españoles en las diferentes áreas. Los contenidos completos de MatEmESp y otra información relevante sobre su proceso de construcción son de libre acceso en www.matemesp.org.

Entre los agentes incluidos en MatEmESp se encuentran los plaguicidas. Este trabajo describe el proceso de estimación seguido y la información disponible sobre la exposición laboral a plaguicidas en MatEmESp. 


\section{MATERIAL Y MÉTODOS}

Para las estimaciones de exposición a plaguicidas en MatEmESp se siguió un proceso con tres etapas. En primer lugar, se identificaron las ocupaciones expuestas a plaguicidas en España en el periodo cubierto por la matriz (1996-2005). A continuación, se seleccionó un grupo de plaguicidas en forma de sustancias activas representativas de la exposición laboral en dicho periodo (agentes de referencia). Por último, se estimó la prevalencia e intensidad de exposición laboral a los agentes seleccionados para cada una de las ocupaciones expuestas consideradas. Se incluyó también una estimación del grado de confianza asignado por el evaluador (JV) en cada una de las estimaciones. Cada una de estas etapas fue precedida de una búsqueda bibliográfica específica, incluyendo artículos científicos, otras matrices empleo-exposición, informes técnicos y otras fuentes de literatura gris.

\section{Identificación de ocupaciones expuestas}

La identificación de las ocupaciones expuestas a plaguicidas en MatEmESp se basó inicialmente en las ocupaciones consideradas expuestas a plaguicidas en la matriz FINJEM. Para ello se establecieron las equivalencias correspondientes entre la codificación de ocupaciones de FINJEM (sistema finlandés a tres dígitos, con 311 categorías) y la de MatEmESp (CNO-94 a cuatro dígitos, con 482 categorías). Esta etapa se llevó a cabo por dos evaluadores independientes. Los resultados de los dos evaluadores se compararon y las ocupaciones en las que existió desacuerdo se discutieron hasta alcanzar el consenso. La revisión de la bibliografía llevó a la identificación de otras ocupaciones expuestas no consideradas originalmente en FINJEM, con las que al final se creó el listado definitivo de las ocupaciones consideradas como expuestas a plaguicidas para población española en MatEmESp.

\section{Selección de agentes de referencia}

Ante la imposibilidad de abarcar la totalidad de sustancias activas autorizadas para su uso durante el periodo de estudio (1996-2005), se seleccionaron aquellas representativas de la exposición laboral a plaguicidas en España durante este periodo. La selección de estos agentes de referencia se llevó a cabo en base al consumo, la relevancia toxicológica, la situación legal de su utilización en España y la existencia de límites de exposición profesional (VLA).

\section{Estimaciones de exposición (intensidad y prevalencia)}

Finalmente, se estimaron las características de la exposición laboral a los agentes de referencia seleccionados en las ocupaciones consideradas como expuestas en términos de prevalencia (proporción de trabajadores expuestos a cada agente en cada ocupación) e intensidad (concentración media en el ambiente de trabajo de la sustancia activa medida en partes por millón). Para ello se utilizaron fundamentalmente datos procedentes de otras MEE, en particular FINJEM, TEMPEST y MATPHYTO, estudios e informes localizados en la bibliografía y los resultados disponibles de un reducido número de mediciones ambientales en empresas españolas y extranjeras. Estas mediciones fueron obtenidas tanto de un servicio de prevención ajeno de ámbito nacional como de evaluaciones nacionales e internacionales publicadas en forma de artículos.

La intensidad de exposición inhalatoria se categorizó en cuatro niveles en función de los VLA disponibles en España para cada agente: bajo ( $\leq 5 \% \mathrm{VLA})$, medio (6-25\%VLA), alto (26-100\%VLA) y muy alto (>100\%VLA). La prevalencia de exposición (proporción de trabajadores expuestos a cada agente en cada ocupación) se estimó como "nula» cuando su frecuencia no supera el 5\%. Así pues, la prevalencia de exposición para cada uno de los agentes considerados se categorizó en tres categoría: baja (5-15\% de los trabajadores expuestos en la ocupación correspondiente), media (15-40\%) y alta (>40\%). 
Por último, junto a cada estimación de exposición, tanto de prevalencia como de intensidad, el evaluador (JV) incluyó una valoración del grado de confianza en el estimador utilizando una escala de tres niveles (alto, medio o bajo). Este es un procedimiento utilizado habitualmente en otras MEE y seguido para otros agentes incluidos en MatEmESp. El grado de confianza en cada estimación es función de la procedencia, cantidad y relevancia de la información disponible. Las estimaciones basadas en la revisión de la literatura disponible, se les asignó un grado bajo de confianza mientras que a aquellas procedentes de otras matrices se les asignó un grado medio. Solo a las estimaciones que pudieron apoyarse en mediciones realizadas en empresas tanto a nivel nacional como en otros países se les asignó un grado de confianza alto.

En MatEmESp se incluyen las estimaciones de exposición obtenidas así como información sobre las fuentes específicas utilizadas para estimación.

\section{RESULTADOS}

La Tabla I contiene las sustancias activas seleccionadas como referencia del periodo de estudio, incluyendo información sobre su situación legal en España así como sus VLA establecidos.

Tabla I. Sustancias activas seleccionadas como referencia durante el periodo de estudio (1996-2005) en el proceso de estimación de las características de la exposición laboral a plaguicidas en trabajadores españoles (matriz empleo-exposición española, proyecto MatEmESp)

\begin{tabular}{lcccc}
\hline \multicolumn{1}{c}{ Clasificación de plaguicidas } & \multicolumn{2}{c}{ Observaciones } \\
\hline Uso & Grupo químico & Sustancia activa & Características & VLA*(VD***) \\
\hline Insecticida & Organoclorado & Endosulfán & Prohibido desde $2006^{1}$ & $0,1 \mathrm{mg} / \mathrm{m}^{3}$ (VD) \\
\hline Insecticida & Organofosforado & Clorpirifós & $\begin{array}{c}\text { En uso como fitosanitario } \\
\text { en } 2005^{2} \text {. Autorizado } \\
\text { como biocida hasta } 2008^{4}\end{array}$ & $0,1 \mathrm{mg} / \mathrm{m}^{3}$ (VD) \\
\hline Insecticida & Carbamato & Metomilo & En uso en $2005^{2}$ & $2,5 \mathrm{mg}^{2} \mathrm{~m}^{3}$ \\
\hline Insecticida & Otros (Piretrinas) & Piretrina & En uso en $2005^{2}$ & $1 \mathrm{mg} / \mathrm{m}^{3}$ \\
\hline Fungicidas & Carbamato & Tiram & En uso en $2005^{2}$ & $1 \mathrm{mg} / \mathrm{m}^{3}$ \\
\hline Fungicidas & Otros (Phtalimida) & Captan & En uso en $2005^{2}$ & $5 \mathrm{mg} / \mathrm{m}^{3}$ \\
\hline Herbicida & A (Bipiridilo) & Dicuat & En uso en $2005^{2}$ & $0,5 \mathrm{mg} / \mathrm{m}^{3}$ (VD) \\
\hline Herbicida & B (Acido Fenoxiacético) & 2,4 D & En uso en $2005^{2}$ & $10 \mathrm{mg} / \mathrm{m}^{3}$ \\
\hline Herbicida & C (Triazina) & Atrazina & Prohibido desde $2004^{3}$ & $5 \mathrm{mg} / \mathrm{m}^{3}$ \\
\hline Herbicida & D (Urea) & Diurón & En uso en $2005^{2}$ & $10 \mathrm{mg} / \mathrm{m}^{3}$ \\
\hline
\end{tabular}

* Valor Límite Ambiental (Límites de exposición profesional para agentes químicos en España, INSHT 2012).

*** VD: Vía dérmica

1) Reglamento (EC) No $777 / 2006$

2) Lista comunitaria de sustancias incluidas, excluidas y en evaluación comunitaria. Ministerio de Medio Ambiente y Medio Rural y Marino, 26 de Abril de 2012.

3) Reglamento (EC) No $775 / 2004$.

4) Decisión de la Comisión Europea de 14 de agosto de 2007.

Las ocupaciones identificadas como expuestas a plaguicidas se presentan en la Tabla II. De este listado $(n=45)$, sólo 14 se consideraban como expuestas a plaguicidas en FINJEM, mientras que las 31 restantes fueron identificadas como ocupaciones expuestas para población laboral española a través de otras fuentes. Para los agentes de referencia en fungicidas y herbicidas, se estimó que en 39 de las ocupaciones expuestas el nivel o intensidad de la exposición era bajo ( $\leq 5 \%$ VLA) y en 6 se estimaron niveles medios de 
exposición (6-25\% VLA). En ningún caso se estimaron ocupaciones expuestas a niveles altos o muy altos para estos agentes. En cuanto a insecticidas de referencia, en 19 ocupaciones se estimó un nivel de exposición bajo, mientras que en las 26 restantes se estimó un nivel de exposición medio. Tampoco se encontraron ocupaciones con niveles de exposición altos o muy altos para estos agentes.

Tabla II. Listado de ocupaciones (CNO-94) para las que se ha considerado existencia de exposición a plaguicidas en la matriz empleo-exposición española (MatEmESp) para el periodo 1996-2005 ( $n=45$ )*

\begin{tabular}{|c|c|c|c|}
\hline Código (CNO-94) & Definición & Código (CNO-94) & Definición \\
\hline 1401 & $\begin{array}{l}\text { Gerencia de explotaciones } \\
\text { agrarias, de caza, de pesca y de } \\
\text { silvicultura con menos de } 10 \\
\text { asalariados (1) }\end{array}$ & 3113 & Asesores agrícolas y forestales \\
\hline 1701 & $\begin{array}{l}\text { Gerencia de explotaciones } \\
\text { agrarias, de caza, de pesca y de } \\
\text { silvicultura sin asalariados (1) }\end{array}$ & 6021 & $\begin{array}{l}\text { Trabajadores cualificados por } \\
\text { cuenta ajena en actividades } \\
\text { agrícolas, excepto en huertas, } \\
\text { viveros y jardines }\end{array}$ \\
\hline 6011 & $\begin{array}{l}\text { Trabajadores cualificados por } \\
\text { cuenta propia en actividades } \\
\text { agrícolas, excepto en huertas, } \\
\text { viveros y jardines (1) }\end{array}$ & 8530 & $\begin{array}{l}\text { Operadores de maquinaria } \\
\text { agrícola móvil }\end{array}$ \\
\hline 6012 & $\begin{array}{l}\text { Trabajadores cualificados por } \\
\text { cuenta propia en huertas, viveros } \\
\text { y jardines (1) }\end{array}$ & 9430 & Peones agropecuarios \\
\hline 6022 & $\begin{array}{l}\text { Trabajadores cualificados por } \\
\text { cuenta ajena en huertas, viveros y } \\
\text { jardines (1) }\end{array}$ & 9800 & $\begin{array}{l}\text { Peones del transporte y } \\
\text { descargadores }\end{array}$ \\
\hline 9410 & Peones agrícolas (1) & 8159 & $\begin{array}{l}\text { Otros operadores de instalaciones } \\
\text { de tratamiento de productos } \\
\text { químicos }\end{array}$ \\
\hline 8040 & $\begin{array}{l}\text { Encargados de taller de madera y } \\
\text { jefes de equipo en la fabricación } \\
\text { de papel (1) }\end{array}$ & 7250 & $\begin{array}{l}\text { Personal de limpieza de fachadas } \\
\text { de edificios y deshollinadores }\end{array}$ \\
\hline 8141 & $\begin{array}{l}\text { Operadores de serrerías, de } \\
\text { máquinas de contrachapado y } \\
\text { de instalaciones conexas para el } \\
\text { tratamiento de la madera (1) }\end{array}$ & 9121 & $\begin{array}{l}\text { Personal de limpieza de oficinas, } \\
\text { hoteles (camareras de piso) y otros } \\
\text { establecimientos similares }\end{array}$ \\
\hline 6241 & $\begin{array}{l}\text { Taladores, trozadores y otros } \\
\text { trabajadores forestales (1) }\end{array}$ & 3123 & Higienistas \\
\hline 6242 & $\begin{array}{l}\text { Carboneros de carbón vegetal y } \\
\text { asimilados (1) }\end{array}$ & 2130 & Veterinarios \\
\hline 9440 & Peones forestales (1) & 3122 & Ayudantes de veterinaria \\
\hline 6220 & $\begin{array}{l}\text { Trabajadores cualificados por } \\
\text { cuenta propia en actividades } \\
\text { forestales y asimilados (1) }\end{array}$ & 3112 & $\begin{array}{l}\text { Técnicos agrónomos, zootécnicos } \\
\text { y forestales }\end{array}$ \\
\hline 8152 & $\begin{array}{l}\text { Operadores en instalaciones de } \\
\text { tratamiento químico térmico (1) }\end{array}$ & 6111 & $\begin{array}{l}\text { Trabajadores cualificados por } \\
\text { cuenta propia en actividades } \\
\text { ganaderas, incluidas las de } \\
\text { animales de compañía y animales } \\
\text { domésticos de piel valiosa }\end{array}$ \\
\hline 8050 & $\begin{array}{l}\text { Jefes de equipo en instalaciones } \\
\text { de tratamiento químico (1) }\end{array}$ & 6112 & $\begin{array}{l}\text { Trabajadores cualificados por } \\
\text { cuenta propia en actividades } \\
\text { avícolas }\end{array}$ \\
\hline
\end{tabular}




\begin{tabular}{|c|c|c|c|}
\hline Código (CNO-94) & Definición & Código (CNO-94) & Definición \\
\hline 8142 & $\begin{array}{l}\text { Operadores en instalaciones para } \\
\text { la fabricación de pasta de papel }\end{array}$ & 6119 & $\begin{array}{l}\text { Otros trabajadores cualificados } \\
\text { por cuenta propia en actividades } \\
\text { ganaderas }\end{array}$ \\
\hline 8143 & $\begin{array}{l}\text { Operadores en instalaciones para } \\
\text { la fabricación de papel }\end{array}$ & 6121 & $\begin{array}{l}\text { Trabajadores cualificados por } \\
\text { cuenta ajena en actividades } \\
\text { ganaderas, incluidas las de } \\
\text { animales de compañía y animales } \\
\text { domésticos de piel valiosa }\end{array}$ \\
\hline 9700 & $\begin{array}{l}\text { Peones de industrias } \\
\text { manufactureras }\end{array}$ & 6122 & $\begin{array}{l}\text { Trabajadores cualificados por } \\
\text { cuenta ajena en actividades } \\
\text { avícolas }\end{array}$ \\
\hline 7911 & $\begin{array}{l}\text { Trabajadores del tratamiento de la } \\
\text { madera }\end{array}$ & 6129 & $\begin{array}{l}\text { Otros trabajadores cualificados } \\
\text { por cuenta ajena en actividades } \\
\text { ganaderas }\end{array}$ \\
\hline 8240 & $\begin{array}{l}\text { Encargado de operadores de } \\
\text { máquinas para fabricar productos } \\
\text { de madera }\end{array}$ & 6210 & $\begin{array}{l}\text { Trabajadores cualificados por } \\
\text { cuenta propia en actividades } \\
\text { agropecuarias }\end{array}$ \\
\hline 8340 & $\begin{array}{l}\text { Operadores de máquinas para } \\
\text { fabricar productos de madera }\end{array}$ & 6230 & $\begin{array}{l}\text { Trabajadores cualificados por } \\
\text { cuenta ajena en actividades } \\
\text { agropecuarias }\end{array}$ \\
\hline 2711 & $\begin{array}{l}\text { Profesionales en ciencias } \\
\text { biológicas y asimilados }\end{array}$ & 9420 & Peones ganaderos \\
\hline 2712 & $\begin{array}{l}\text { Ingenieros técnicos en } \\
\text { especialidades agrícolas y } \\
\text { forestales }\end{array}$ & 3112 & $\begin{array}{l}\text { Técnicos agrónomos, zootécnicos } \\
\text { y forestales }\end{array}$ \\
\hline 2113 & Agrónomos y asimilados & & \\
\hline
\end{tabular}

1) Estas ocupaciones fueron consideradas expuestas a plaguicidas tanto en MatEmESp como en FINJEM $(n=14)$. El resto de ocupaciones fueron consideradas expuestas en MatEmESp, pero no en FINJEM ( $n=31$ ) (ver descripción del proceso en el texto).

Las Tablas III, IV y V muestran las ocupaciones para las que se identificaron los mayores indicadores de exposición en términos de prevalencia e intensidad según los diferentes grupos de agentes seleccionados. Tanto en fungicidas como en herbicidas (Tablas III y IV), se encontraron las mayores prevalencias de exposición $(>15 \%$ de trabajadores expuestos en la ocupación) y las mayores intensidades de exposición (con valores entre el 6 y el 25\% del VLA) en 6 ocupaciones agrícolas. En esta actividad destaca la exposición a 2,4 D, Captam y Diurón, con prevalencias altas (en algunos casos más del $90 \%$ de trabajadores expuestos) e intensidades de exposición inhalatoria entre 0,06 y $0,20 \mathrm{ppm}$. En cuanto a los insecticidas (Tabla V), las mayores prevalencias de exposición se estimaron para las mismas 6 ocupaciones anteriores y en otras 27 asociadas a actividades de ganadería, silvicultura, jardinería, transporte, inspección y limpieza, fabricación de plaguicidas y tratamiento de la madera. Destacan aquí sustancias activas como el Metomilo, en actividades como la agricultura, la jardinería y la silvicultura, con elevadas proporciones de trabajadores expuestos (50-82\%) e intensidades de exposición inhalatoria entre 0,06 y $0,10 \mathrm{ppm}$. 
Tabla III. Ocupaciones con mayores indicadores de exposición a los fungicidas de referencia (prevalencia de trabajadores expuestos $\geq 15 \%$ y niveles de exposición del 6-25\% del VLA**). Matriz empleo-exposición española (MatEmESp)

\begin{tabular}{|c|c|c|c|c|c|}
\hline $\begin{array}{l}\text { Código* } \\
\text { CNO-94 }\end{array}$ & $\begin{array}{c}\text { Descripción } \\
\text { de la ocupación }\end{array}$ & P (\%) Tiram & I (ppm) Tiram & P (\%) Captam & I (ppm) Captam \\
\hline 6011 & $\begin{array}{l}\text { Trabajadores } \\
\text { cualificados por } \\
\text { cuenta propia en } \\
\text { actividades agrícolas, } \\
\text { excepto en huertas, } \\
\text { viveros y jardines }\end{array}$ & 38 & 0,02 & 98 & 0,06 \\
\hline 6012 & $\begin{array}{l}\text { Trabajadores } \\
\text { cualificados por } \\
\text { cuenta propia en } \\
\text { huertas, viveros y } \\
\text { jardines }\end{array}$ & 38 & 0,02 & 98 & 0,06 \\
\hline 6021 & $\begin{array}{l}\text { Trabajadores } \\
\text { cualificados por } \\
\text { cuenta ajena en } \\
\text { actividades agrícolas, } \\
\text { excepto en huertas, } \\
\text { viveros y jardines }\end{array}$ & 38 & 0,02 & 98 & 0,06 \\
\hline 9410 & Peones agrícolas & 38 & 0,02 & 98 & 0,06 \\
\hline 8530 & $\begin{array}{l}\text { Operadores de } \\
\text { maquinaria agrícola } \\
\text { móvil }\end{array}$ & 38 & 0,02 & 98 & 0,06 \\
\hline 9430 & Peones agropecuarios & 38 & 0,02 & 98 & 0,06 \\
\hline
\end{tabular}

$\mathrm{P}=$ Prevalencia de exposición (\% de trabajadores expuestos) I=Intensidad de exposición (concentración, en ppm, de sustancia activa en el aire).

* CNO-94: Clasificación Nacional de Ocupaciones 1994.

**:VLA: Valor Límite Ambiental (INSHT 2012).

Tabla IV. Ocupaciones con mayores indicadores de exposición a los herbicidas de referencia (prevalencia de trabajadores expuestos $\geq 15 \%$ y niveles de exposición del $6-25 \%$ del VLA**). Matriz empleo-exposición española (MatEmESp)

\begin{tabular}{clcccc}
\hline $\begin{array}{l}\text { Código* } \\
\text { CNO-94 }\end{array}$ & Descripción de la ocupación & P (\%) Atrazina & I (ppm) Atrazina & P (\%) Dicuat & I (ppm) Dicuat \\
\hline 6011 & $\begin{array}{l}\text { Trabajadores cualificados } \\
\text { por cuenta propia en } \\
\text { actividades agrícolas, } \\
\text { excepto en huertas, viveros } \\
\text { y jardines }\end{array}$ & 34 & 0,10 & 32 & 0,01 \\
\hline 6012 & $\begin{array}{l}\text { Trabajadores cualificados } \\
\text { por cuenta propia en } \\
\text { huertas, viveros y jardines }\end{array}$ & 34 & 0,10 & 32 & 0,01 \\
\hline 6021 & $\begin{array}{l}\text { Trabajadores cualificados } \\
\text { por cuenta ajena en } \\
\text { actividades agrícolas, } \\
\text { excepto en huertas, viveros } \\
\text { y jardines }\end{array}$ & 34 & 0,10 & 32 & 0,01 \\
\hline 9410 & Peones agrícolas & 34 & & \\
\hline 8530 & $\begin{array}{l}\text { Operadores de maquinaria } \\
\text { agrícola móvil }\end{array}$ & 34 & 0,10 & 32 & 0,01 \\
\hline 9430 & Peones agropecuarios & 34 & 0,10 & 32 \\
\hline
\end{tabular}




\begin{tabular}{clcccc}
\hline $\begin{array}{l}\text { Código* } \\
\text { CNO-94 }\end{array}$ & Descripción de la ocupación & P (\%) 2,4 D & I (ppm) 2,4 D & P (\%) Diuron & I (ppm) Diuron \\
\hline 6011 & $\begin{array}{l}\text { Trabajadores cualificados } \\
\text { por cuenta propia en } \\
\text { actividades agrícolas, } \\
\text { excepto en huertas, viveros } \\
\text { y jardines }\end{array}$ & 70 & 0,20 & 90 & 0,20 \\
\hline $\begin{array}{l}\text { Trabajadores cualificados } \\
\text { por cuenta propia en } \\
\text { huertas, viveros y jardines }\end{array}$ & 70 & 0,20 & 90 & 0,20 \\
\hline 6021 & $\begin{array}{l}\text { Trabajadores cualificados } \\
\text { por cuenta ajena en } \\
\text { actividades agrícolas, } \\
\text { excepto en huertas, viveros } \\
\text { y jardines }\end{array}$ & 70 & 0,20 & 90 & 0,20 \\
\hline 9410 & Peones agrícolas & 70 & 0,20 & 90 & 0,20 \\
\hline 8530 & $\begin{array}{l}\text { Operadores de maquinaria } \\
\text { agrícola móvil }\end{array}$ & 70 & 0,20 & 90 & 0,20 \\
\hline 9430 & Peones agropecuarios & 70 & 0,20 & 90 & 0 \\
\hline
\end{tabular}

$\mathrm{P}=$ Prevalencia de exposición (\% de trabajadores expuestos) I=Intensidad de exposición (concentración, en ppm, de sustancia activa en el aire).

* CNO-94: Clasificación Nacional de Ocupaciones 1994.

**VLA: Valor Límite Ambiental (INSHT 2012).

Tabla V. Ocupaciones con mayores indicadores de exposición a los insecticidas de referencia (prevalencia de trabajadores expuestos $\geq 15 \%$ y niveles de exposición del 6-25\% del VLA**). Matriz empleo-exposición española (MatEmESp)

\begin{tabular}{|c|c|c|c|c|c|c|c|c|c|}
\hline $\begin{array}{l}\text { Código* } \\
\text { CNO-94 }\end{array}$ & Descripción de la ocupación & $\begin{array}{l}\text { P (\%) } \\
\text { Clor. }\end{array}$ & $\begin{array}{l}\text { I (ppm) } \\
\text { Clor. }\end{array}$ & $\begin{array}{l}\text { P (\%) } \\
\text { End. }\end{array}$ & $\begin{array}{c}\text { I (ppm) } \\
\text { End. }\end{array}$ & $\begin{array}{l}\text { P (\%) } \\
\text { Met. }\end{array}$ & $\begin{array}{c}\text { I (ppm) } \\
\text { Met. }\end{array}$ & $\begin{array}{l}\text { P (\%) } \\
\text { Piret. }\end{array}$ & $\begin{array}{c}\text { I (ppm) } \\
\text { Piret. }\end{array}$ \\
\hline 3123 & Higienistas & 15 & 0,001 & - & - & 70 & 0,06 & - & - \\
\hline 6011 & $\begin{array}{l}\text { Trabajadores cualificados por } \\
\text { cuenta propia en actividades } \\
\text { agrícolas, excepto en huertas, } \\
\text { viveros y jardines }\end{array}$ & 88 & 0,001 & - & - & - & - & 90 & 0,01 \\
\hline 6012 & $\begin{array}{l}\text { Trabajadores cualificados por } \\
\text { cuenta propia en huertas, } \\
\text { viveros y jardines }\end{array}$ & 88 & 0,001 & - & - & 50 & 0,06 & 99 & 0,01 \\
\hline 6021 & $\begin{array}{l}\text { Trabajadores cualificados por } \\
\text { cuenta ajena en actividades } \\
\text { agrícolas, excepto en huertas, } \\
\text { viveros y jardines }\end{array}$ & 88 & 0,001 & - & - & 50 & 0,06 & 99 & 0,01 \\
\hline 6022 & $\begin{array}{l}\text { Trabajadores cualificados } \\
\text { por cuenta ajena en huertas, } \\
\text { viveros y jardines }\end{array}$ & 82 & 0,001 & - & - & 82 & 0,10 & - & - \\
\hline 6111 & $\begin{array}{l}\text { Trabajadores cualificados por } \\
\text { cuenta propia en actividades } \\
\text { ganaderas, incluidas las de } \\
\text { animales de compañía y } \\
\text { animales domésticos de piel } \\
\text { valiosa }\end{array}$ & 40 & 0,001 & - & - & - & - & 60 & 0,01 \\
\hline 6112 & $\begin{array}{l}\text { Trabajadores cualificados por } \\
\text { cuenta propia en actividades } \\
\text { avícolas }\end{array}$ & 40 & 0,001 & - & - & - & - & 60 & 0,01 \\
\hline
\end{tabular}




\begin{tabular}{|c|c|c|c|c|c|c|c|c|c|}
\hline $\begin{array}{l}\text { Código* } \\
\text { CNO-94 }\end{array}$ & Descripción de la ocupación & $\begin{array}{l}\text { P (\%) } \\
\text { Clor. }\end{array}$ & $\begin{array}{l}\text { I (ppm) } \\
\text { Clor. }\end{array}$ & $\begin{array}{l}P(\%) \\
\text { End. }\end{array}$ & $\begin{array}{l}\text { I (ppm) } \\
\text { End. }\end{array}$ & $\begin{array}{l}\text { P (\%) } \\
\text { Met. }\end{array}$ & $\begin{array}{l}\text { I (ppm) } \\
\text { Met. }\end{array}$ & $\begin{array}{l}\text { P (\%) } \\
\text { Piret. }\end{array}$ & $\begin{array}{l}\text { I (ppm) } \\
\text { Piret. }\end{array}$ \\
\hline 6119 & $\begin{array}{l}\text { Otros trabajadores } \\
\text { cualificados por cuenta } \\
\text { propia en actividades } \\
\text { ganaderas }\end{array}$ & 40 & 0,001 & - & - & - & - & 60 & 0,01 \\
\hline 6121 & $\begin{array}{l}\text { Trabajadores cualificados por } \\
\text { cuenta ajena en actividades } \\
\text { ganaderas, incluidas las de } \\
\text { animales de compañía y } \\
\text { animales domésticos de piel } \\
\text { valiosa }\end{array}$ & 40 & 0,001 & - & - & - & - & 60 & 0,01 \\
\hline 6122 & $\begin{array}{l}\text { Trabajadores cualificados por } \\
\text { cuenta ajena en actividades } \\
\text { avícolas }\end{array}$ & 40 & 0,001 & - & - & - & - & 60 & 0,01 \\
\hline 6129 & $\begin{array}{l}\text { Otros trabajadores } \\
\text { cualificados por cuenta ajena } \\
\text { en actividades ganaderas }\end{array}$ & 40 & 0,001 & - & - & - & - & 60 & 0,01 \\
\hline 6210 & $\begin{array}{l}\text { Trabajadores cualificados por } \\
\text { cuenta propia en actividades } \\
\text { agropecuarias }\end{array}$ & 40 & 0,001 & - & - & - & - & 60 & 0,01 \\
\hline 6220 & $\begin{array}{l}\text { Trabajadores cualificados por } \\
\text { cuenta propia en actividades } \\
\text { forestales y asimilados }\end{array}$ & 50 & 0,001 & - & - & 70 & 0,06 & - & - \\
\hline 6230 & $\begin{array}{l}\text { Trabajadores cualificados por } \\
\text { cuenta ajena en actividades } \\
\text { agropecuarias }\end{array}$ & 40 & 0,001 & - & - & - & - & 60 & 0,01 \\
\hline 6241 & $\begin{array}{l}\text { Taladores, trozadores y otros } \\
\text { trabajadores forestales }\end{array}$ & - & - & - & - & 70 & 0,06 & - & - \\
\hline 6242 & $\begin{array}{l}\text { Carboneros de carbón } \\
\text { vegetal y asimilados }\end{array}$ & - & - & - & - & 70 & 0,06 & - & - \\
\hline 7250 & $\begin{array}{l}\text { Personal de limpieza de } \\
\text { fachadas de edificios y } \\
\text { deshollinadores }\end{array}$ & 15 & 0,001 & - & - & 70 & 0,06 & - & - \\
\hline 7911 & $\begin{array}{l}\text { Trabajadores del tratamiento } \\
\text { de la madera }\end{array}$ & 33 & 0,05 & 33 & 0,05 & 33 & 0,05 & - & - \\
\hline 8040 & $\begin{array}{l}\text { Encargados de taller de } \\
\text { madera y jefes de equipo en } \\
\text { la fabricación de papel }\end{array}$ & 33 & 0,05 & 33 & 0,05 & 33 & 0,05 & - & - \\
\hline 8050 & $\begin{array}{l}\text { Jefes de equipo en } \\
\text { instalaciones de tratamiento } \\
\text { químico }\end{array}$ & 43 & 0,001 & - & - & - & - & - & - \\
\hline 8141 & $\begin{array}{l}\text { Operadores de serrerías, de } \\
\text { máquinas de contrachapado } \\
\text { y de instalaciones conexas } \\
\text { para el tratamiento de la } \\
\text { madera }\end{array}$ & 33 & 0,05 & 33 & 0,05 & 33 & 0,05 & - & - \\
\hline 8152 & $\begin{array}{l}\text { Operadores en instalaciones } \\
\text { de tratamiento químico } \\
\text { térmico }\end{array}$ & 43 & 0,001 & - & - & - & - & - & - \\
\hline 8159 & $\begin{array}{l}\text { Otros operadores de } \\
\text { instalaciones de tratamiento } \\
\text { de productos químicos }\end{array}$ & 43 & 0,001 & - & - & - & - & - & - \\
\hline
\end{tabular}




\begin{tabular}{|c|c|c|c|c|c|c|c|c|c|}
\hline $\begin{array}{l}\text { Código* } \\
\text { CNO-94 }\end{array}$ & Descripción de la ocupación & $\begin{array}{l}\text { P (\%) } \\
\text { Clor. }\end{array}$ & $\begin{array}{l}\text { I (ppm) } \\
\text { Clor. }\end{array}$ & $\begin{array}{l}\mathbf{P}(\%) \\
\text { End. }\end{array}$ & $\begin{array}{l}\text { I (ppm) } \\
\text { End. }\end{array}$ & $\begin{array}{l}\text { P (\%) } \\
\text { Met. }\end{array}$ & $\begin{array}{l}\text { I (ppm) } \\
\text { Met. }\end{array}$ & $\begin{array}{l}\text { P (\%) } \\
\text { Piret. }\end{array}$ & $\begin{array}{l}\text { I (ppm) } \\
\text { Piret. }\end{array}$ \\
\hline 8240 & $\begin{array}{l}\text { Encargado de operadores } \\
\text { de máquinas para fabricar } \\
\text { productos de madera }\end{array}$ & 33 & 0,05 & 33 & 0,05 & 33 & 0,05 & - & - \\
\hline 8340 & $\begin{array}{l}\text { Operadores de máquinas } \\
\text { para fabricar productos de } \\
\text { madera }\end{array}$ & 33 & 0,05 & 33 & 0,05 & 33 & 0,05 & - & - \\
\hline 8530 & $\begin{array}{l}\text { Operadores de maquinaria } \\
\text { agrícola móvil }\end{array}$ & 88 & 0,001 & - & - & 50 & 0,06 & - & - \\
\hline 9121 & $\begin{array}{l}\text { Personal de limpieza } \\
\text { de oficinas, hoteles } \\
\text { (camareras de piso) y otros } \\
\text { establecimientos similares }\end{array}$ & 15 & 0,001 & - & - & 70 & 0,06 & - & - \\
\hline 9410 & Peones agrícolas & 88 & 0,001 & - & - & 50 & 0,06 & - & - \\
\hline 9420 & Peones ganaderos & 40 & 0,001 & - & - & - & - & 60 & 0,01 \\
\hline 9430 & Peones agropecuarios & 88 & 0,001 & - & - & 50 & 0,06 & 60 & 0,01 \\
\hline 9440 & Peones forestales & - & - & - & - & 70 & 0,06 & - & - \\
\hline 9700 & $\begin{array}{l}\text { Peones de industrias } \\
\text { manufactureras (fabricación } \\
\text { plaguicidas) }\end{array}$ & 43 & 0,001 & 33 & 0,05 & 33 & 0,05 & - & - \\
\hline 9800 & $\begin{array}{l}\text { Peones del transporte y } \\
\text { descargadores }\end{array}$ & 43 & 0,001 & - & - & - & - & - & - \\
\hline
\end{tabular}

$\mathrm{P}=$ Prevalencia de exposición (\% de trabajadores expuestos) I=Intensidad de exposición (concentración, en ppm, de sustancia activa en el aire) *Insecticidas: Clor.=Clorpirifos; End.=Endosulfan; Met.=Metomilo; Piret.= Piretrina.

* CNO-94: Clasificación Nacional de Ocupaciones 1994.

**VLA: Valor Límite Ambiental (INSHT 2012).

Respecto a la fiabilidad del total de las estimaciones para la intensidad de exposición incluidas en la matriz ( $n=324)$, el evaluador (JV) asignó un grado medio de confianza (estimaciones basadas fundamentalmente en datos procedentes de otras matrices) a 220, mientras que a 76 se les asignó un grado de confianza bajo (estimaciones basadas solo en fuentes bibliográficas). En 28 de las estimaciones el evaluador consideró que el grado de confianza era alto (estimaciones basadas en datos de otras matrices apoyadas en mediciones de campo). Respecto a las estimaciones de prevalencia de exposición, 248 se basaron en datos de otras matrices (grado de confianza medio), mientras que 76 fueron establecidas en base a la bibliografía consultada (grado de confianza bajo).

Los resultados completos de todo el proceso de estimación de la exposición a plaguicidas incluyendo ocupaciones expuestas, agentes de referencia, estimaciones de exposición, fuentes de información, grado de confianza y otros datos de interés) se encuentra disponible en la página web de MatEmESp (www.matemesp.org).

\section{DISCUSIÓN}

Este trabajo supone el primer esfuerzo de recolección sistemática de datos relacionados con la exposición laboral a plaguicidas en población trabajadora en España para su organización en forma de una matriz empleo-exposición. Se ha estimado que las ocupaciones con indicadores más elevados de exposición a fungicidas y herbicidas pertenecen a actividades de agricultura, en las que es probable que exista una mayor frecuencia de exposición, puesto que en estas ocupaciones se utilizan más del 90\% de los plaguicidas a nivel nacional ${ }^{2,3}$. En otras ocupaciones, como las asociadas a actividades de 
ganadería, silvicultura o tratamiento de la madera, se han estimado índices de exposición altos sólo para los agentes pertenecientes a la familia de los insecticidas. En este mismo grupo de agentes, en ocupaciones asociadas a actividades de higiene ambiental y limpieza, se estimaron niveles medios tanto de intensidad como de prevalencia de exposición.

La matriz empleo-exposición finlandesa (FINJEM) ha sido utilizada con cierta frecuencia como base para establecer la exposición laboral a distintos agentes en estudios sobre población trabajadora en España ${ }^{16,17}$ y es también el punto de partida por defecto para las estimaciones de exposición incluidas en MatEmESp. En el proceso aquí descrito, se identificaron 31 ocupaciones con exposición laboral a plaguicidas en España que, sin embargo, no aparecen como ocupaciones expuestas a estos agentes en FINJEM. Es de esperar que existan diferencias notables en el uso de plaguicidas en Finlandia y España, por ejemplo, por diferencias secundarias a la climatología, a las características de su uso en el sector primario o a la existencia de empresas fabricantes de estas sustancias. Pero estas diferencias pueden existir también en relación con otros agentes y exposiciones laborales. Este hecho debe tenerse en consideración al utilizar matrices generadas en otros países para estudios epidemiológicos sobre población laboral española.

En este trabajo se ha seguido el procedimiento habitual para la construcción de matrices empleo-exposición, basado en fuentes bibliográficas, mediciones ambientales y criterio experto. Sin embargo, la disponibilidad de datos procedentes de mediciones ambientales de exposición laboral a plaguicidas en España ha sido muy limitada. En conjunto, solo se identificaron mediciones con información sobre niveles puntuales o medios de exposición laboral a distintos agentes en diez situaciones de trabajo y sólo cinco de ellas referidas a trabajadores españoles. En particular, solamente se localizaron mediciones de campo pertenecientes a las actividades de fabricación, higiene ambiental e invernaderos. Todo ello contribuyó a que el porcentaje del grado de confianza en las estimaciones establecidas sea en su mayoría medio o bajo. Por otro lado, aunque es posible que búsquedas bibliográficas más exhaustivas permitieran localizar un mayor número de estudios publicados con mediciones de exposición, las fuentes más importantes de estas mediciones en España son los servicios de prevención propios y ajenos. Sin embargo, la obtención de estas mediciones para usos científicos o de gestión sigue siendo un problema pendiente de resolver. En especial, dada la intensa actividad de los servicios de higiene industrial en empresas de todo el territorio nacional, sería de especial interés desarrollar un sistema de homogeneización y centralización de los datos disponibles que permitiera un tratamiento conjunto de la información para éste u otros fines, siguiendo el modelo que ya aplican organismos nacionales de salud laboral como el instituto de salud laboral finlandés, FIOH ${ }^{18}$, o algunas iniciativas descritas en Canadá ${ }^{19}$.

Adicionalmente, hay que tener en cuenta que las mediciones ambientales en los lugares de trabajo suelen proceder de campañas para el control del cumplimiento de los límites normativos, por lo que su representatividad respecto a las características generales de exposición en todos los trabajadores de cada ocupación es incierta. Las fuentes bibliográficas consultadas proceden en su mayoría de estudios y datos disponibles para otros países, aunque se ha procurado, siempre mediante criterio experto, identificar las situaciones que pudieran considerarse más similares a la realidad de las condiciones de los trabajadores españoles. Estas limitaciones, bien conocidas y compartidas con otros trabajos similares ${ }^{20}$, deben siempre considerarse en el uso de la información contenida en las MEE.

En este mismo sentido, las estimaciones de exposición incluidas en las matrices empleo-exposición suponen una aproximación a las condiciones del colectivo, por su incapacidad para cubrir toda la variabilidad individual en la exposición de los trabajadores en una misma ocupación en función de las características de la empresa, las tareas desarrolladas, las prácticas de prevención y protección en el lugar de trabajo, etc. La información proporcionada por las matrices debe entenderse en términos de probabilidad de exposición dada la pertenencia a un colectivo (ocupación). Esto resulta útil para la investigación epidemiológica, como ha demostrado el uso de matrices en relación con distintos problemas de salud laboral ${ }^{21}$, y también para priorizar y planificar la prevención 
de riesgos laborales en las empresas, un uso menos habitual de las matrices pero que consideramos igualmente interesante ${ }^{22}$.

En el proceso de estimación de la exposición laboral a plaguicidas seguido en este trabajo no ha sido posible obtener información suficiente acerca de ocupaciones en determinados sectores con exposición potencial, pero desconocida. Tal es el caso de sectores como la alimentación, el textil, la manufactura de aparatos electrónicos, la depuración de aguas o la acuicultura ${ }^{1}$. La información incluida en MatEmEsp es la mejor disponible en el formato de matriz empleo-exposición para población laboral española. Aun así, dicha información sigue siendo mejorable, y sería de interés dedicarle los recursos necesarios para la revisión y actualización periódica de la información a partir de la disponibilidad de nuevos datos.

Aunque la prohibición en España de los plaguicidas más peligrosos ha supuesto una reducción de los riesgos para la salud humana y el medio ambiente, la autorización de nuevas sustancias activas, de efectos muchas veces desconocidos, hace necesaria la vigilancia y evaluación continua de estos compuestos, tanto con criterios individuales como poblacionales, referidos estos últimos a la identificación y control de los colectivos de trabajadores con mayores probabilidades de exposición. Los datos contenidos en MatEmESp son un primer paso, limitado pero imprescindible, para sistematizar la vigilancia poblacional de la exposición laboral a plaguicidas.

\section{AGRADECIMIENTOS}

Nuestro agradecimiento a M. ${ }^{a}$ Carmen González Galarzo (CiSAL) por su colaboración en la codificación de las ocupaciones y la gestión del conjunto de datos en MatEmESp, así como a Miguel Ángel Alba (Sociedad de Prevención de FREMAP) por proporcionar datos procedentes de mediciones ambientales a plaguicidas en distintos ambientes de trabajo en España.

\section{FINANCIACIÓN}

Este trabajo se llevó a cabo gracias a la financiación recibida por el autor principal (JV) del Centro de Investigación en Salud Laboral (CiSAL) de la Universitat Pompeu Fabra (UPF) en Barcelona para la realización en este centro del Máster en Salud Laboral. El proyecto MatEmESp ha recibido financiación del Instituto Sindical de Trabajo, Ambiente y Salud (ISTAS), del Centro de Investigación en Salud Laboral (CiSAL), del Instituto de Salud Carlos III (referencia PI081496), de la Conselleria de Sanitat de la Generalitat Valenciana (referencia 066/2009), del Instituto Nacional de Seguridad e Higiene en el Trabajo y de la Fundación Prevent.

\section{BIBLIOGRAFÍA}

1. Ramírez, J. A., Lacasaña, M. Plaguicidas: clasificación, uso, toxicología y medición de la exposición. Arch Prev Riesg Labor 2001;4(2):67-75.

2. Vila, J. Desarrollo de una matriz empleo-exposición (MEE) para describir la exposición de los trabajadores a plaguicidas en España (1996-2005). Trabajo Fin de Máster de Salud Laboral del Centro de Investigación en Salud Laboral. Barcelona: Universitat Pompeu Fabra [online] 2012 [citado 05 Octubre 2014], 56 pág. Disponible en la World Wide Web: http://www.istas.ccoo.es/matemesp/Memoria_TFM_Definitiva_J_Vila. pdf

3. AEPLA. Memoria 2000-2010: Construyendo el futuro de la agricultura. Asociación Empresarial para la Protección de las Plantas [online]; 2011 [citado 05 Octubre 2014], 52 pág. Disponible en la World Wide Web: http://www.aepla.es/publicaciones/69-aepla-2000-2010-construyendo-el-futuro-de-la-agricultura

4. Ministerio Medio Ambiente. Resultados Encuesta de Comercialización 2012. En: Estadística anual de consumo de productos fitosanitarios en la Agricultura. Ministerio Medio Ambiente [online]. 2012 
[citado 05 Octubre 2014], 15 pág. Disponible en la World Wide Web: http://www.magrama.gob.es/es/ estadistica/temas/estadisticas-agrarias/agricultura/estadisticas-medios-produccion/fitosanitarios.aspx

5. Ministerio de Trabajo. Encuesta Nacional de Condiciones de Trabajo en el Sector Agropecuario. INSHT [online]; 2009 [citado 05 Octubre 2014], 175 pág. Disponible en la World Wide Web: http://www.insht.es/ Observatorio/Contenidos/InformesPropios/Desarrollados/Ficheros/Encuesta\%20Nacional\%20 Agropecuaria.pdf

6. García, A. M., Ramírez, A., Lacasaña, M. Prácticas de utilización de plaguicidas en agricultores. Gac Sanit 2002; 16(3):236-240.

7. Ministerio de Sanidad y Consumo. Protocolos de vigilancia sanitaria específica: Plaguicidas. MSC [online]; 1999 [citado 05 Octubre 2014], 24 pág. Disponible en la World Wide Web: http://www.msssi.gob. es/ciudadanos/saludAmbLaboral/docs/plaguicidas.pdf

8. Delgado-Martínez, M. Efectos crónicos de los fitosanitarios. En: Respuesta ante las intoxicaciones agudas por plaguicidas. Guillén, J., Serrano, J. L., et al. Consejería de Salud de la Junta de Andalucía [online] 2003 [citado 05 Octubre 2014]:87-96. Disponible en la World Wide Web: http://www.juntadeandalucia.es/ salud/export/sites/csalud/galerias/documentos/p_4_p_1_vigilancia_de_la_salud/intoxicaciones_agudas_ plaguicidas.pdf

9. Delgado, P. Riesgos para la salud en plantas de formulación de plaguicidas. 1. a ed. Madrid: Servicio de ediciones y publicaciones del Instituto Nacional de Seguridad e Higiene en el Trabajo (INSHT); 1994.

10. Kauppinen, T., Toikkanen, J., Pukkala, E. From cross-tabulations to multipurpose exposure information systems: a new job-exposure matrix. Am J Ind Med 1998;33(4):409-17.

11. Schinasi, L. S., Beane, W. L., Freeman, L. E., Baldi, I., Kromhout, H., Lebailly, P. Development of a pesticide crop/livestock exposure matrix using data from three agricultural cohort studies in the AGRICOH consortium. Presentación en el congreso EPICOH 2013, Utrecht, Holanda: Occup Environ Med 2013; 70(Suppl 1):A44.

12. Févotte, J., Dananché, B., Delabre, L., Ducamp, S., Garras, L., Houot, M. et al. Matgéné: a program to develop job-exposure matrices in the general population in France, Ann Occup Hyg. 2011 Oct;55(8):865-78.

13. Dick, F., Semple, S., van Tongeren, M., Miller, B., Ritchie, P., Sherriff, D., Cherrie, W. Development of a Task-Exposure Matrix (TEM) for Pesticide Use (TEMPEST). Ann Hyg 2010(54):443-452.

14. García, A. M., González-Galarzo, M. C., Kauppinen, T., Delclos, G. L., Benavides, F. G. A Job-Exposure Matrix for Research and Surveillance of Occupational Health and Safety in Spanish Workers: MatEmESp. Am J Ind Med. 2013 Jul 1. doi: 10.1002/ajim.22213

15. García, A. M., González-Galarzo, M. C. La matriz empleo-exposición española: MatEmESp. Arch Prev Riesgos Labor 2012;15(3):121-123.

16. Santibáñez, M., Vioque, J., Alguacil, J., De la Hera, M. G., Moreno-Osset, E., Carrato, A., et al. Occupational exposures and risk of pancreatic cancer. Eur J Epidemiol. 2010;25(10):721-30.

17. Santibañez, M., Alguacil, J., de la Hera, M. G., Navarrete-Muñoz, E. M., Llorca, J., Aragonés, N., et al. Occupational exposures and risk of stomach cancer by histological type. Occup Environ Med. 2012;69(4):268-75.

18. Kauppinen, T. Finnish Occupational Exposure Databases. Appl Occ Env Hyg 2001;16(2):154-158.

19. Lavoué. J., Gérin, M., Bégin, D., Ostiguy, C., Arcand, R., Adib, G. Valorisation des données d'exposition professionnelle mesurées au Québec depuis 1980 par les équipes du Réseau public québécois en santé au travail - Étude préliminaire. Études et recherches. Informe R-723, Montreal: Institut de recherche RobertSauvé en santé et en sécurité du travail (IRSST) [online], 2012 [citado 05 Octubre 2014], 80 pág. Disponible en la World Wide Web: http://www.irsst.qc.ca/media/documents/PubIRSST/R-723.pdf

20. Lavoué, J., Pintos, J., van Tongeren, M., Kincl, L., Richardson, L., Kauppinen, T., et al. Comparison of exposure estimates in the Finnish job-exposure matrix FINJEM with a JEM derived from expert assessments performed in Montreal. Occup Environ Med 2012;69(7):465-71.

21. Coughlin, S. S., Chiazze, L. Jr. Job-exposure matrices in epidemiologic research and medical surveillance. Occup Med. 1990;5(3):633-46.

22. García, A. M., González-Galarzo, M. C., Alba, M. A., Gordo, J., van der Haar, R., Briceño, F., Gadea, R., López, M., Benavides, F. G. Proyecto MatEmESp: matrices empleo-exposición para trabajadores españoles. Seguridad y Medio Ambiente. 2011: (123):22-34 